\title{
EFECTO DE LA APLICACIÓN DE ÁCIDO NAFTALÉN ACÉTICO SOBRE EL ENRAIZAMIENTO DE ESTACAS LEÑOSAS DE Croton urucurana Baill., Tabernaemontana catharinensis A. DC. Y Paullinia elegans Cambess
}

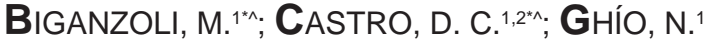 \\ Gabriel, P. M. ${ }^{1} ;$ Álvarez, N. ${ }^{1}$ \& BuyatTI, M. A. ${ }^{1}$
}

\section{RESUMEN}

El objetivo de este trabajo fue evaluar el enraizamiento de estacas de Croton urucurana Baill., Tabernaemontana catharinensis A. DC. y Paullinia elegans Cambess conocidas en Argentina como "Sangre de drago" (SD), "Horquetero" (TC) y "Ojo de muñeca" (PE), respectivamente. Se recolectaron 200 estacas leñosas por especie; una vez defoliadas se aplicó ácido naftalén acético (ANA) en las siguientes dosis: 0, 1000, 2500 y $3500 \mathrm{mg} . \mathrm{L}^{-1}$ en SD y TC y $0,500,1000$ y $2500 \mathrm{mg} . \mathrm{L}^{-1}$ en PE. Se evaluó la formación y calidad de raíces y el diámetro de las estacas a la altura del cuello (DAC). En SD las dosis de ANA $\geq 1000 \mathrm{mg} \cdot \mathrm{L}^{-1}$ aumentaron la probabilidad de enraizamiento de 0,36 a 0,71 . En PE hubo baja probabilidad de enraizamiento $(0,13)$ independientemente de la dosis de ANA, pero aumentó en función del DAC. No se observó formación de raíces en TC. La calidad de enraizamiento no fue afectada por la diferentes dosis de ANA, pero sí el DAC en SD.

Key words: ácido naftalénacético, ANA, ácido indolbutírico, IBA, propagación vegetativa, promotores de enraizamiento.

1.- Facultad de Ciencias Agrarias. Universidad Nacional del Litoral. Kreder 2805, Esperanza (3080), provincia de Santa Fe, Argentina.

2.- Consejo Nacional de Investigaciones Científicas y Técnicas

* Autor para correspondencia: dcastro@fca.unl.edu.ar

$\wedge$ El primer y segundo autor contribuyeron igualmente a este trabajo.

Manuscrito recibido el 14 de agosto de 2020 y aceptado para su publicación el 24 de agosto de 2020 .

Biganzoli, M.; Castro, D.C.; Ghio, N.; Gabriel, P.M.; Álvarez, N; Buyatti, M.A. Efecto de la aplicación de ácido naftalén acético sobre el enraizamiento de estacas leñosas de Croton urucurana Baill., Tabernaemontana catharinensis A. DC. y Paullinia elegans Cambess. FAVE - Ciencias Agrarias 20 (1): 189-203.

CC BY-NC-SA 4.0 


\begin{abstract}
Effect of NAA concentrations on rooting of hardwood cuttings of Croton urucurana Baill., Tabernaemontana catharinensis A. DC. and Paullinia elegans Cambess.

The objective of this work was to evaluate the rooting potential of "Sangre de Drago" (SD: Croton urucurana Baill.), "Horquetero" (TC: Tabernaemontana catharinensis A. DC.) and "Ojo de muñeca" (PE: Paullinia elegans Cambess.) cuttings by immersion in 1-Naphthaleneacetic acid (NAA). Two hundred cuttings without leaves per species were collected and immersed in 0, 1000, 2500 and $3500 \mathrm{ppm}$ of NAA (for SD and TC) and 0,500, 1000 and $2500 \mathrm{ppm}$ of NAA for PE. At the end of the rooting period the following variables were registered on each cutting; the presence of roots, diameter at the base of the cutting (DAC) and rooting quality. Rooting probability was highest (0.76) for NAA than control (0.36) on SD cuttings. The rooting probability was very low on PE regardless of the NAA dose (0.13) but was directly proportional to the DAC. Root formation was not observed on TC, regardless of the NAA dose nor DAC. Rooting quality was unaffected by NAA dose but highly related to DAC in SD.
\end{abstract}

Key words: naftalen acetic acid, indolbutiric acid, IBA, vegetative propagation, softwood cuttings, quick deep.

\section{INTRODUCCIÓN}

La producción mundial de plantas ornamentales y sus industrias subsidiarias generan anualmente un valor agregado aproximado de entre 250 a 400000 millones de dólares, alrededor del 0,5\% del producto bruto mundial (Mata et al. 2013). Para Argentina se estima que el valor bruto de la producción está entre 220 y 240 millones de dólares (Morisigue, D. 2011). En cuanto a la diversidad biológica, los recursos genéticos nativos y los mercados globales asociados a ello, forman la base de una proporción significativa de la actividad económica mundial. Una estimación de las ventas de los mercados globales anuales, para el área de los productos hortícolas ornamentales derivados completamente de recursos genéticos, se encuentra entre 16 y 19 billones de dólares (Kate y Laird, 2000).
En Argentina el desarrollo comercial de recursos fitogenéticos nativos se centró en especies de hábito herbáceo, mientras que las leñosas (géneros Tecoma y Handroanthus híbridos interespecíficos entre Jacaranda mimosifolia D. Don y J. cuspidifolia Mart), se desarrollaron para obtener floración temprana y porte compacto para uso en macetas (Soto et al. 2011). Sin embargo, existen otras especies con alto valor paisajístico para arbolado urbano o jardines, que actualmente no se encuentran desarrolladas económicamente como Croton urucurana, Taberbaemontana catharinensis (ambas de porte arbóreo) y Paullinia elegans (de tipo apoyante).

Para el desarrollo económico de especies ornamentales, la propagación vegetativa es un aspecto crucial ya que permite clonar individuos con superioridad en su valor paisajístico (atractivo ornamental, tolerancia a 
estrés, adaptabilidad a ambientes restrictivos, etc.) (Dole y Gibson 2006). Dentro de las ventajas más importantes que presenta este método se encuentran la producción de clones, la superación de problemas de la propagación sexual (baja viabilidad, baja energía germinativa, baja producción de semillas, etc.) y la ausencia del período juvenil en las plantas propagadas vegetativamente (Hartmann et al. 2002). En el género Paullinia existen evidencias de especies con semillas recalcitrantes como $P$. pinnata L. (Francis 2004) y P. cupana Kunth "Guaraná" (de Carvalho et al. 1982), por lo tanto la propagación vegetativa representa una oportunidad para ampliar el período productivo, además de mantener clones. Esta situación también se observó en $P$. elegans (Ghio et al. 2018). En T. catharinensis no existen problemas relacionados a la propagación sexual, no obstante es interesante estudiar la propagación vegetativa con el objetivo de evitar el período juvenil de esta especie, dado que su principal atractivo es su floración. En C. urucurana debe realizarse una selección de la semilla por color para aumentar el porcentaje de germinación y además las semillas se deben someter a alternancia de temperatura (Scalon et al. 2012). En este caso la propagación vegetativa podría ser una técnica más sencilla que la propagación sexual.

El éxito comercial de la reproducción asexual por estacas depende de factores endógenos y exógenos que influyen en el potencial de enraizamiento de las estacas. Dentro de los factores exógenos, uno de los más importantes es la utilización de auxinas sintéticas que se aplican en concentraciones de 500 a 10000 ppm dependiendo del grado de lignificación de la estaca y la presencia o ausencia de hojas (Dole y
Gibson 2006). Otro factor importante es el tiempo de inmersión en la solución que va desde segundos a días, dependiendo de la concentración utilizada. El Ácido Naftalén Acético (ANA) es una auxina sintética barata, potente y móvil, por lo que su uso requiere de un ajuste preciso de la dosis y el tiempo de inmersión (Hartmann et al. 2002). Dentro de los factores endógenos, la especie tiene gran relevancia. La bibliografía muestra resultados inconsistentes en el enraizamiento de especies del género Croton que dependen de la posición de la estaca utilizada en la rama (apical, sub-apical, basal), de la presencia de hojas, la inclinación de la estaca en cámara de enraizamiento, del tipo de sustrato utilizado y el tipo y dosis de hormona (Chaves 2016; da Gama et al. 2015; Osakada y Yuyama 2008; Santos et al. 2011). Sumado a esto, antecedentes dentro de la misma familia ( $E u$ phorbiaceae) muestran que dosis de 3000 ppm de AIB y 2500 ppm de ANA inducen el enraizamiento y elevan la uniformidad en la producción de raíces (Dole y Gibson 2006). En cuanto al género Tabernaemontana la bibliografía muestra que dosis de 3000 a 5000 ppm de AIB indujeron un alto porcentaje de enraizamiento en estacas semi-leñosas con hojas de T. coronaria, una especie de fácil enraizamiento (Rawat et al. 2014). Por otro lado, los antecedentes en el género Paullinia muestran resultados contradictorios en el enraizamiento de estacas herbáceas con la utilización de AIB entre 0 y 6000 ppm, siendo el factor determinante el clon (Albertino et al. 2012; Arruda et al. 2007; Atroch et al. 2011; Rodrigues y Lucchesi 1987). Trabajos previos muestran que en la familia Sapindaceae existe una falta de respuesta de estacas a promotores de enraizamiento (Amorim et al. 2019). 
Por lo mencionado anteriormente, el objetivo de este trabajo fue evaluar el potencial de enraizamiento y la calidad de raíces en estacas en Croton urucurana, Paullinia elegans y Tabernaemontana catharinensis sometidas a diferentes dosis de ANA.

\section{MATERIALES Y MÉTODOS}

\section{Material vegetal y área de recolección de muestras}

Los propágulos de "Sangre de drago" (C. urucurana), "Horquetero" (T. catharinensis) y "Ojo de muñeca" (P. elegans) se recolectaron en diferentes áreas de su distribución natural, en los departamentos La Capital, Juan de Garay y San Javier de la provincia de Santa Fe, en los primeros días del mes de Julio del año 2017 (Figura 1). Las especies mencionadas fueron seleccionadas en función de su valor paisajístico representado por su floración, fructificación, follaje, bajos requerimientos de mantenimiento y aptitud para el uso en parques, jardines y/o arbolado urbano de alineación.

Para cada una de las tres especies, se colectaron 200 estacas caulinares, leñosas, homogéneas, de 15 centímetros de longitud aproximadamente, sin tener en cuenta su posición a lo largo del ramo, diámetro de la estaca, ni la posición del ramo en las plantas madres. La tarea de recolección comenzó durante las primeras horas de la mañana. Los propágulos de cada especie se extrajeron de 8 a 10 plantas adultas y en estado silvestre. Estos genotipos se seleccionaron visualmente teniendo en cuenta a aquellos de mejor comportamiento fitosanitario.
Se procedió a la extracción de las estacas, realizando un corte recto en la base, dejando un nudo inmediatamente por encima del mismo y un corte en bisel en el extremo apical. También se llevó a cabo el deshojado de las estacas. Para todos los cortes se utilizaron tijeras correctamente desinfectadas en una solución de hipoclorito de sodio al $50 \% \mathrm{v} / \mathrm{v}$. Las estacas se desinfectaron realizando dos enjuagues en una solución de hipoclorito de sodio $8 \%(\mathrm{v} / \mathrm{v})$, posteriormente, se colocaron en bolsas con cierre hermético, identificando cada genotipo y se almacenaron en conservadora termoeléctrica portátil Coleman $\AA$ en condiciones de baja temperatura $2 \pm 3,8^{\circ} \mathrm{C}$ y $90 \%$ de HR hasta la aplicación de hormonas.

\section{Tratamientos hormonales y ambiente de propagación}

Antes de la inmersión en ANA se efectuó un nuevo lavado del material mediante la inmersión de las estacas en solución de agua con hipoclorito de sodio al $8 \%(\mathrm{v} / \mathrm{v})$ durante diez minutos. Luego, se realizó una inmersión en fungicida sistémico Carbendazim (50\% SC- suspensión concentrada) a una concentración de $3 \mathrm{ml} . \mathrm{L}^{-1}$ durante el mismo tiempo. Se formaron grupos de 50 estacas con aproximadamente la misma proporción de los genotipos recolectados, para eliminar el efecto genotipo sobre el enraizamiento. A cada uno de estos grupos se le asignó al azar uno de los tratamientos hormonales que se muestran en la tabla 1 . Dentro de cada grupo, cada estaca fue individualizada con un número progresivo empezando en el 1 en el primer tratamiento y terminando en 200 en el último tratamiento de cada especie. 


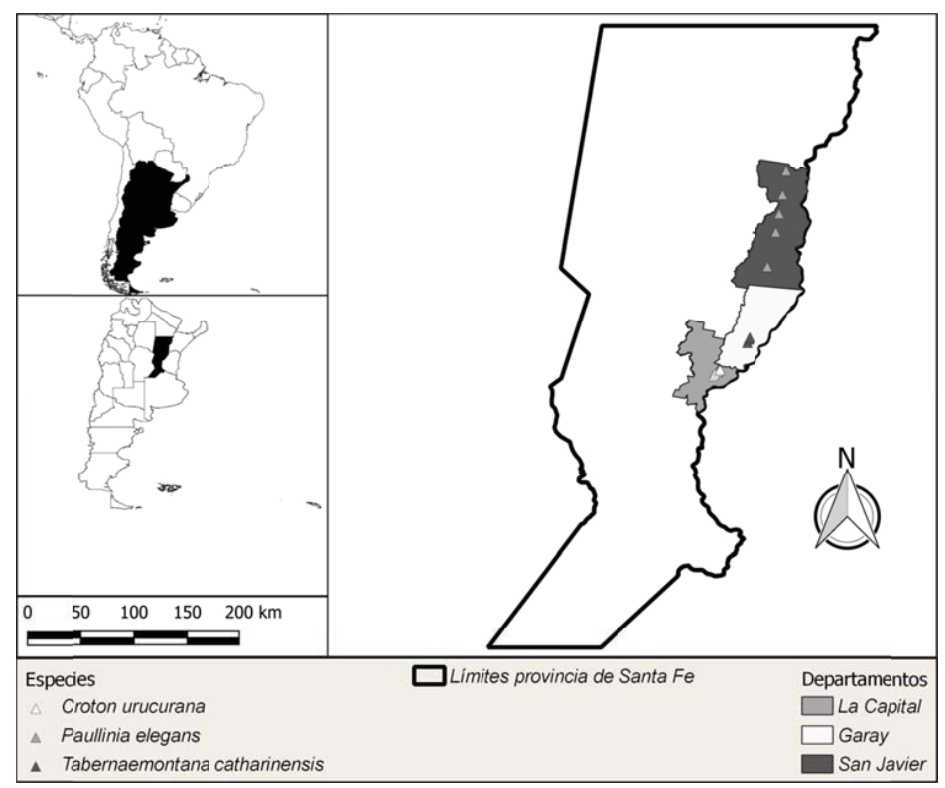

Figura 1. Ubicación de los puntos de colecta de estacas de "Sangre de Drago" (Croton urucurana), "Ojo de muñeca" (Paullinia elegans) y "Horquetero" (Tabernaemontana catharinensis) en la provincia de Santa Fe, Argentina.

Figure 1. Sampling points of cuttings from "Sangre de Drago" (Croton urucurana), "Ojo de muñeca" (Paullinia elegans) and "Horquetero" (Tabernaemontana catharinensis) in Santa Fe province, Argentina

Tabla 1. Tratamientos hormonales realizados a cada especie y número de estacas por tratamiento. Table 1. NAA dose per species (Concentración de ANA mg/L) and number of cuttings per dose $\left(N^{o}\right.$ de estacas pore specie).

\begin{tabular}{|c|c|c|c|c|c|c|}
\hline & \multicolumn{7}{|c|}{ Concentración de ANA (mg.L $\left.{ }^{-1}\right)$} & $\begin{array}{c}N^{\circ} \text { de Estacas } \\
\text { por especie }\end{array}$ \\
\hline & 0 & 500 & 1000 & 2500 & 3500 & \\
\hline C. urucurana & 50 & 0 & 50 & 50 & 50 & 200 \\
\hline T. catharinensis & 50 & 0 & 50 & 50 & 50 & 200 \\
\hline P. elegans & 50 & 50 & 50 & 50 & 0 & 200 \\
\hline
\end{tabular}


Para los tratamientos hormonales se usó como hormona enraizante al ácido naftalenacético (ANA), sal sódica, pureza 99\%, hidrosoluble, marca Dalton $\AA$. Aunque la sal es hidrosoluble, en algunas concentraciones se observaba un residuo sólido de la sal que fue disuelto con hidróxido de potasio $(\mathrm{KOH}) 1 \mathrm{~N}$, en cantidades suficientes y se llevó al volumen deseado con agua destilada. El tiempo de inmersión en la solución hormonal fue de 5 segundos, independientemente de la concentración utilizada. Se optó por el ANA por su mayor actividad hormonal y por su menor costo, en comparación con el AIB, teniendo presente la futura utilización por parte de viveristas.

En una primera instancia las estacas se llevaron a cámara de crecimiento a temperatura controlada $\left(22 \pm 1{ }^{\circ} \mathrm{C}\right)$, y alta HR $(90 \%)$ para inducir la formación del callo, etapa de "callusing" según Dole y Gibson (2006). Para ello se utilizaron bandejas de $30 \times 60 \times 20 \mathrm{~cm}$, con perlita agrícola como sustrato, la que previamente fue esterilizada en estufa a $60^{\circ} \mathrm{C}$ durante 3 días y humedecida. Para mantener elevada la HR, cada bandeja fue tapada con bolsas de polietileno negro. Esta etapa tuvo una duración de 60 días durante la cual se monitoreó y registró la aparición de raíces en cada estaca y la presencia de enfermedades, y se realizaron aplicaciones preventivas de Captan.

Una vez formado el callo y detectado el desarrollo inicial de las raíces (largo $\geq$ $1 \mathrm{~mm}$ ), las estacas fueron trasladadas a un invernadero de dimensiones $3 \times 3 \times 3 \mathrm{~m}$ para su crecimiento posterior. En esta etapa de crecimiento de raíces, las estacas fueron colocadas en tubetes numerados de 250 $\mathrm{cm}^{3}$ dispuestos en bandejas de 54 unidades, donde se utilizó el sustrato MultiPro ${ }^{\mathrm{TM}}$ de la marca GrowMix ${ }^{\circledR}$, compuesto por turba de musgo Sphagnun spp. de fibras finas, compost de corteza fina, perlita, corrector de $\mathrm{pH}$ y fertilizantes. Se instaló un sistema de riego automático (15 minutos de riego por hora, con un volumen de $12 \mathrm{~L} / \mathrm{h})$. Se registró la temperatura y HR del ambiente en invernadero a través de datalogger i-Button ${ }^{\circledR}$ (modelo Thermochron. Rango: - 40 a $85{ }^{\circ} \mathrm{C}$ y 0 a $100 \%$ HR. Resolución: $0,1{ }^{\circ} \mathrm{C}$ y $0,5 \% \mathrm{HR}$; error: $+/-0,5^{\circ} \mathrm{C}$ y $+/-3 \% \mathrm{HR}$ ) cada 15 minutos. Esta etapa tuvo una duración de 45 días. La duración del experimento completo fue de 105 días.

\section{Variables respuesta analizadas}

Una vez finalizado el experimento se registró el enraizamiento de cada estaca (presencia o ausencia de raíces) y el diámetro de las estacas a la altura del cuello (DAC). Por otro lado, se evaluaron las variables de calidad de enraizamiento (longitud total de raíces, diámetro medio de la raíz y volumen de raíces) mediante el software WinRhi$\mathrm{zo}^{\mathrm{TM}}$ (2016) y un escáner compatible. Para ello se extrajo el total de la masa radicular de aquellas estacas que enraizaron satisfactoriamente y se las conservó en recipientes individuales con FAA (formaldehido, ácido acético, alcohol $96^{\circ}$ y agua destilada, en una relación $2: 1: 10: 7)$. Para cada especie se utilizó un diseño completamente aleatorizado con 4 tratamientos y 50 repeticiones por tratamiento, siendo la unidad experimental una estaca. Los datos de enraizamiento se analizaron mediante el ajuste de Modelos Lineales Generalizados y regresiones logísticas con la función $\mathrm{glm}$ del paquete stats del lenguaje de programación estadística R (R Development Core Team 2017), utilizando la función de enlace logit. Dichos modelos tuvieron la forma $\mathrm{PE}=\mathrm{ANA}+$ $\mathrm{DAC}+\mathrm{ANA}^{*} \mathrm{DAC}+\varepsilon$, donde PE es la probabilidad de enraizamiento, ANA es el 
efecto de la aplicación de ANA, DAC es el efecto del DAC y ANA*DAC es el efecto de la interacción sobre la probabilidad de enraizamiento, mientras que $\varepsilon$ es el error del modelo. Cuando los modelos lineales generalizados mostraron efecto significativo del ANA, se realizó la comparación entre niveles de ANA aplicados mediante la función emmeans del paquete emmeans de R (Lenth 2020). La calidad de enraizamiento se analizó mediante el ajuste de regresiones lineales múltiples con la función Im del paquete stats del lenguaje de programación estadística $\mathrm{R}$ ( $\mathrm{R}$ Development Core Team 2017). La forma de los modelos fue la misma que para probabilidad de enraizamiento y se eligieron las variables a retener por el modelo mediante el procedimiento "stepway" utilizando la función ols_step_ both_p del paquete olsrr (Hebbali 2018). Las figuras se realizaron utilizando el paquete ggplot2 de R (Wickham 2016).

\section{RESULTADOS Y DISCUSIÓN}

\section{Efecto de dosis crecientes de ANA en el porcentaje de enraizamiento \\ "Sangre de drago" (C. urucurana)}

La aplicación de ANA a dosis $\geq 1000$ ppm, aumentó a casi el doble la probabilidad de enraizamiento de las estacas ( $p=$ $0,01)$ en comparación con el control (Figura 2). El diámetro a la altura del cuello de la estaca (DAC) no tuvo un efecto significativo sobre la probabilidad de enraizamiento $(\mathrm{p}=0,54)$ y tampoco la interacción DAC $\times$ ANA $(p=0.63)$. Este modelo clasificó correctamente al $70 \%$ de las estacas, como enraizada o no enraizada en función de la dosis de ANA. Como se observa en la Figura 2, las dosis de ANA superiores a 1000 ppm mostraron una diferencia signifi- cativa en la probabilidad de enraizamiento en comparación con el control $(\mathrm{p}<0,05)$, pero no hubo diferencias entre las concentraciones de ANA utilizadas $(\mathrm{p}=0,90)$. Una probabilidad de enraizamiento de $36 \%$ sin la aplicación de hormonas demuestra que C. urucurana es una especie de fácil enraizamiento. Esto concuerda con lo observado por Santos et al. (2011) quienes obtuvieron un $23 \%$ de enraizamiento sin aplicación de hormonas, pero contrasta con lo obtenido por Chaves (2016) quien no observó enraizamiento en el tratamiento control. Otras especies del género Croton mostraron una tasa moderada de enraizamiento (20-30\%) sin la aplicación de hormonas (Amaya et al. 2019; Cunha et al. 2012). De acuerdo con Santos et al (2011) la aplicación de AIB no tuvo efecto significativo en la formación de raíces en $C$. urucurana, posiblemente debido a las bajas dosis aplicadas (100-300 ppm) y a que utilizaron AIB en polvo, una hormona con menor capacidad para inducir raíces que el ANA. Osakada y Yuyama (2008) detectaron un efecto perjudicial de la aplicación de hormonas (200 ppm de ANA y AIB) en estacas de C. lechleri con inmersión durante 14 horas. Sin embargo, en Croton zehntneri la inmersión por 10 segundos en dosis crecientes de AIB desde 0 a 5000 ppm elevó de $20 \%$ a $90 \%$ el porcentaje de enraizamiento (Cunha et al. 2012), demostrando que la aplicación de hormonas es crucial para la viabilidad de la propagación por estacas de Croton a nivel comercial, tal como se demuestra en el presente trabajo. La variabilidad observada en la Figura 2 puede deberse a diversos factores endógenos como el genotipo de donde se obtuvo la estaca, la edad y el nivel nutricional del mismo y, el tipo de estaca, tal como fue demostrado en otras especies como Eucalyptus cloeziana F. Muell. (Al- 


\section{Biganzoli et al.}

meida et al. 2007), Olea europaea (Silva et al. 2012) y Nothofagus alessandrii (Santelices 2005). No obstante, debido al diseño experimental utilizado en nuestro trabajo, no puede separarse del error el componente asociado a los factores antes mencionados.

\section{“Horquetero" (T. catharinensis)}

Los tratamientos realizados en estacas de "Horquetero" no fueron efectivos para inducir su enraizamiento, dado que fue nula la formación de raíces en todos los tratamientos aplicados. No obstante, se pudo observar, que gran parte de las estacas que no emitieron raíz pero que permanecían vivas durante los recuentos, emitieron brotes que murieron al poco tiempo; lo que se debería a que las condiciones ambientales óptimas para el enraizamiento desencadenan la brotación en yemas sin latencia (Chaves 2016). Esta brotación, anterior a la formación de raíces, dificulta el enraizamiento de las estacas debido a que las reservas energéticas son destinadas a la brotación, en lugar de la inducción y crecimiento de raíces, además de provocar desbalances hormonales que también afectan el proceso de enraizamiento (Hartmann et al. 2002).

Los resultados de este trabajo demuestran que T. catharinensis es una especie de difícil enraizamiento a través de la propagación por estacas leñosas sin hojas. Esto contrasta con lo observado en T. coronaria, donde se obtuvo un $90 \%$ de enraizamiento

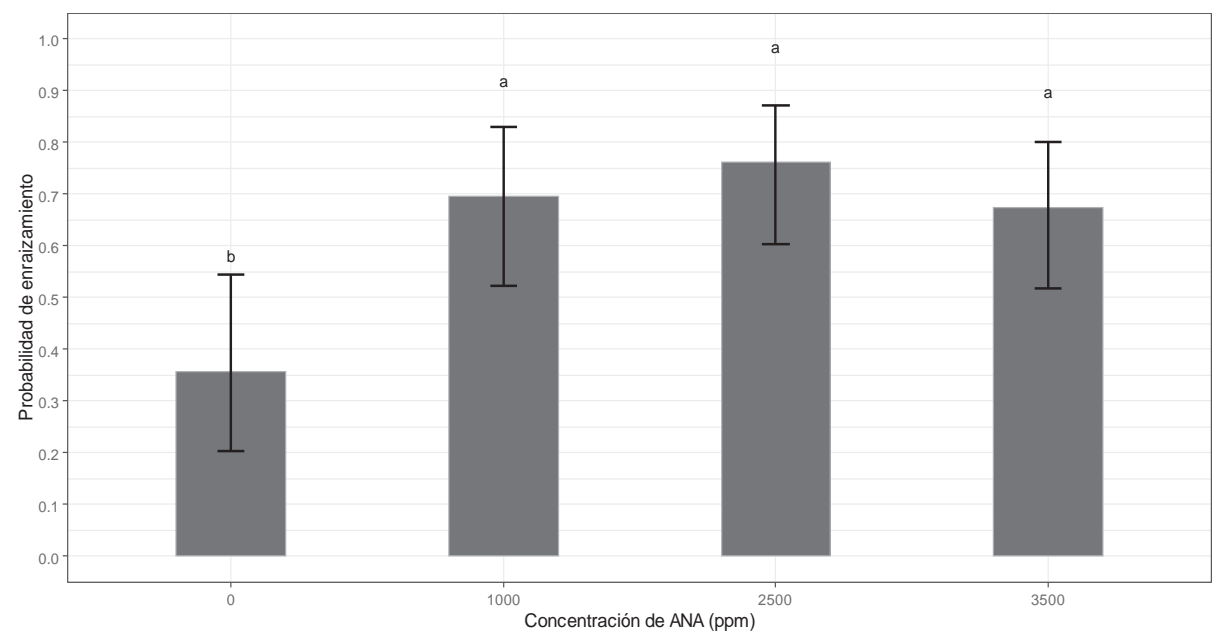

Figura 2. Efecto de la concentración de ácido naftalén acético (ANA) sobre la probabilidad de enraizamiento de estacas de Croton urucurana. Las barras verticales sobre las columnas indican en intervalo de confianza de la probabilidad estimada. Letras distintas indican diferencia estadistica por el test de Tukey.

Figure 2. NAA effect on rooting probability of Croton urucurana cuttings. Vertical bars on each column represent the 95 percent confidence interval for rooting probability. Different letters on each bar indicates statistical diference by Tukey's test. 
de estacas sub-leñosas con hojas sin aplicación de hormonas (Rawat et al. 2014). En esta especie, la inmersión de estacas con hojas de plantas jóvenes en AIB $(\geq 3000$ ppm) por 10 minutos aumentó al $100 \%$ el porcentaje de enraizamiento, y mejoró significativamente el largo, diámetro y número total de raíces. En T. catharinensis, la propagación in vitro de microestacas con hojas a partir de plantas madres jóvenes, produjo un enraizamiento superior al $70 \%$. Dado que las estacas del presente trabajo se mantuvieron vivas durante el experimento es posible que la ausencia de inducción radicular haya sido consecuencia de una baja concentración de hormona exógena, un corto período de inmersión, la utilización de tejido muy lignificado o la selección de plantas adultas con baja capacidad de enraizamiento, factores muy importantes para la inducción radical en estacas (Hartmann et al. 2002).

\section{“Ojo de muñeca” (P. elegans)}

En "Ojo de muñeca" se observó inducción radical en un bajo porcentaje de estacas (13\%), como efecto de la aplicación de ANA, sin diferencia entre las concentraciones $(\mathrm{p}>0,05)$. No obstante, existió una relación positiva entre el DAC y la probabilidad de enraizamiento $(\mathrm{p}=0,0001)$; esta última aumentó 0,30 veces por cada milímetro de aumento en el DAC, observándose valores superiores al $50 \%$ con diámetros mayores a $13 \mathrm{~mm}$. (Figura 3). Por otro lado, no hubo efecto de la interacción DA$\mathrm{C} \times$ ANA $(\mathrm{p}=0.56)$. Este modelo clasificó correctamente al $95 \%$ de las estacas como enraizada o no enraizada en función de su DAC. Esto pone de relevancia que $P$. elegans es una especie de fácil enraizamiento, ya que se indujo la formación de raíces sin utilización de hormonas exógenas en más del $50 \%$ de los casos en los que las estacas tenían un diámetro mayor a $13 \mathrm{~mm}$ (Figura 3). Esto concuerda con lo demostrado para P. cupana donde el enraizamiento de estacas semi-leñosas con o sin hojas y sin aplicación de hormonas va del $30 \%$ al $90 \%$ (Albertino et al. 2012; Arruda et al. 2007; Atroch et al. 2011; Rodrigues y Lucchesi 1987). La relación ascendente entre el DAC y la probabilidad de enraizamiento podría ser una consecuencia de la escasez de reservas para abastecer los cambios anatómicos que se desencadenan luego de la inducción a la formación de raíces, en estacas cuyo DAC es inferior a $13 \mathrm{~mm}$. Esta relación entre el porcentaje y calidad de enraizamiento y el tamaño de la estaca fue demostrada para diversas especies (Dias et al. 2013; Martins et al. 2015; Santos et al. 2011). En cuanto a la aplicación de hormonas exógenas, los antecedentes en $P$. cupana indican que dosis superiores a $50 \mathrm{ppm}$ de AIB inhiben la formación de raíces (Atroch et al. 2011); para esta especie el mayor enraizamiento se produce utilizando estacas con hojas y sometiéndolas a etiolización previo a la disposición en camas de enraizamiento (Rodrigues y Lucchesi 1987). La falta de respuesta de las estacas a promotores exógenos de enraizamiento en el género Paullinia parece ser una característica de la familia Sapindaceae (Amorim et al. 2019) y podría deberse a la presencia de un adecuado balance hormonal para promover el enraizamiento, o a la presencia de raíces adventicias preformadas, como ocurre en otras especies (Hartmann et al. 2002). Nuevos experimentos de enraizamiento con $P$. elegans deberían realizarse en estacas semi-leñosas con DAC superior a $13 \mathrm{~mm}$ y presencia de hojas, o previa etiolización de los ramos que darán origen a las estacas. 


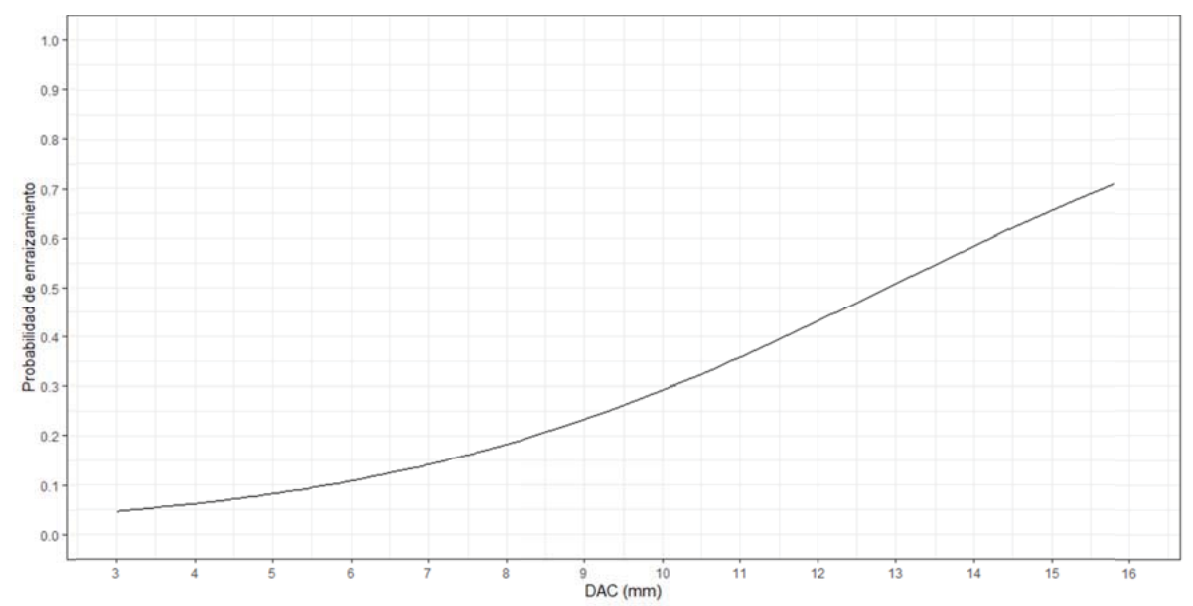

Figura 3. Efecto del diámetro a la altura del cuello de la estaca (DAC) sobre la probabilidad de enraizamiento de estacas leñosas sin hojas de Paullinia elegans.

Figure 3. Cutting diameter (DAC) effect on the rooting probability of Paullinia elegans cuttings.

Efecto de dosis crecientes de ANA en la calidad de enraizamiento

Los datos que se muestran son los de "Sangre de drago" (C. urucurana), ya que en las otras dos especies el enraizamiento provocado por las dosis de ANA fue nulo o despreciable.

\section{Largo total de raíces}

El largo total de raíces fue afectado únicamente por el DAC de la estaca $(\mathrm{p}=$ $0.0051, \mathrm{R}^{2}=0,32$ ). Por cada milímetro de aumento del DAC, el largo total de raíces se incrementó en 1,77 cm (Figura 4). Nuestros datos concuerdan con los de Santos et al. (2011), quienes no observaron un efecto del tratamiento hormonal sobre la calidad de enraizamiento de C. urucurana. Tampoco fue observado un efecto directo de la hormona sobre la calidad de enraizamiento en antecedentes con otras especies forestales (Richmond 1984).
No obstante, en estudios previos se observó una relación directa entre el DAC de la estaca y la calidad de enraizamiento, particularmente en la longitud total de raíces (Santos et al. 2011). Dado que la formación de raíces se produce a partir de las reservas acumuladas en las estacas que aún no tienen hojas, la relación directamente proporcional entre el diámetro de las mismas y el largo total de raíces, indica que estacas con mayor tejido de reserva (mayor diámetro) tienen mayor contenido de energía y nutrientes, que son utilizados para la formación de raíces, lo cual es independiente del nivel de hormonas exógenas aplicadas (Ming 1996).

\section{Diámetro medio de raíces}

El diámetro medio de las raíces no fue influenciado por el tratamiento hormonal ( $p$ $>0,05)$, ni por el DAC ( $p>0,05)$, ni por la interacción DAC*ANA ( $\mathrm{p}>0,05)$. El diámetro medio de las raíces, independientemente del tratamiento hormonal recibido, fue de $0,80 \mathrm{~mm}$.

Revista FAVE - Ciencias Agrarias 20 (1) 2021 


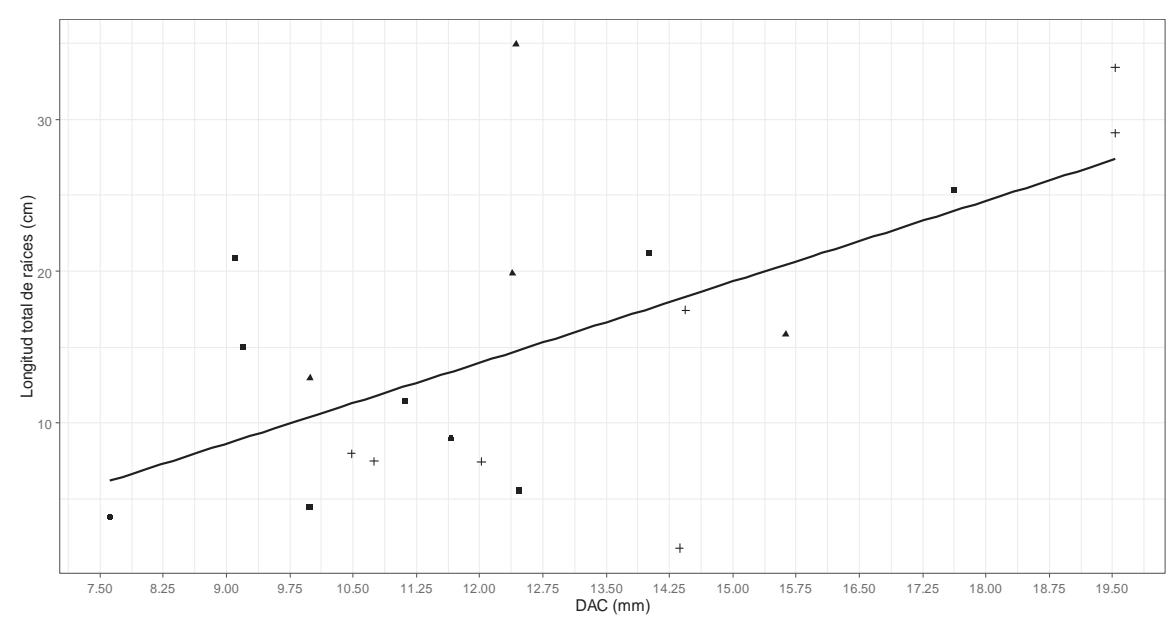

Figura 4. Efecto del diámetro de la estaca (DAC) sobre la longitud total de raíces (LTR) en Cro-

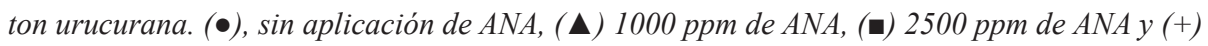
3500 ppm de ANA. LTR = 1,775*DAC-7,314.

Figure 4. Cutting diameter (DAC) effect on total root length (TRL) of Croton urucurana cuttings.

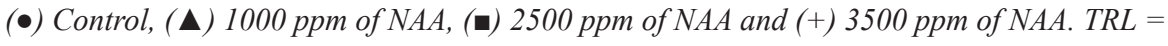
$1.775 * D A C-7.314$.

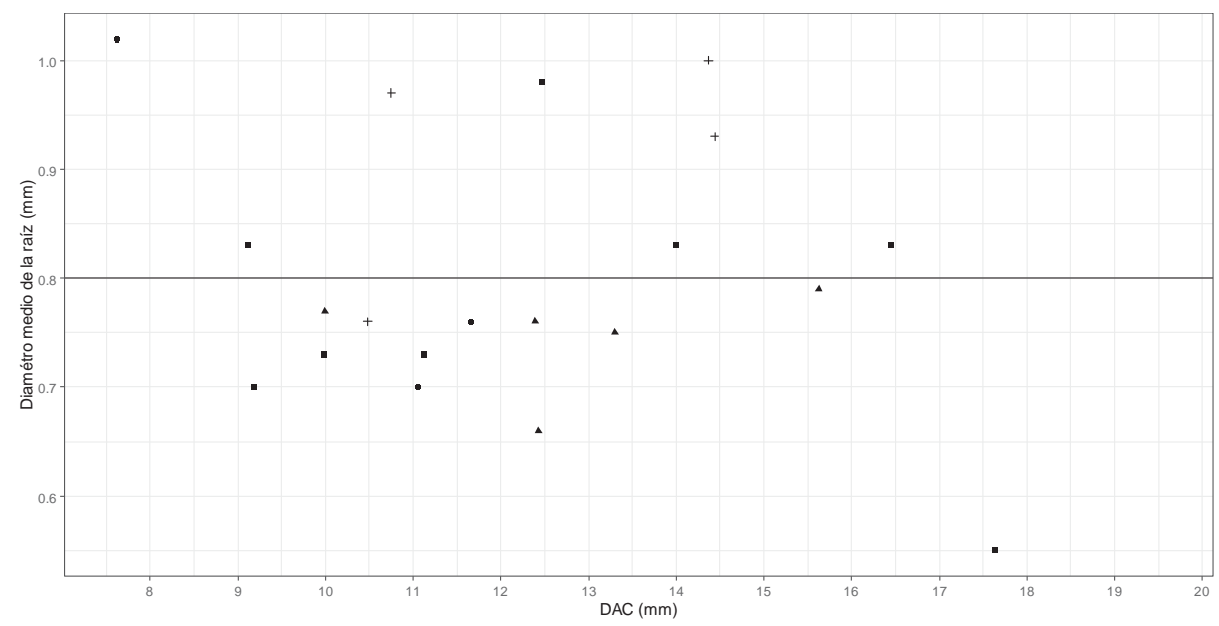

Figura 5. Diámetro medio de raíces en estacas de Croton urucurana. (•) sin aplicación de ANA, (ム) 1000 ppm de ANA, (ロ) 2500 ppm de ANA y (+) 3500 ppm de ANA.

Figure 5. Cutting diameter effect (DAC) on average root diameter (ARD) of Croton urucurana

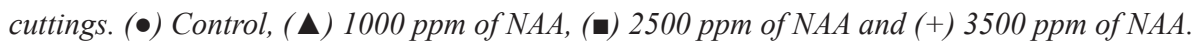
$A R D=0.8 \mathrm{~mm}$ 


\section{Volumen de raíces}

El volumen total de raíces por estaca no fue influenciado por la aplicación de dosis crecientes de ANA $(\mathrm{p}=0,58)$, tampoco por el DAC $(\mathrm{p}=0,30)$, ni por la interacción ANA*DAC $(p=0,35)$. Como se observa en la Figura 6, la gran variabilidad observada en el volumen medio de raíces por estaca impide la detección de diferencias entre tratamientos. El volumen promedio de raíces por estaca, independientemente de la concentración de ANA aplicada y del DAC, fue de $0,066 \mathrm{~mL}$.

\section{CONCLUSIONES}

La especie "Sangre de drago" (Croton urucurana Baill.,) es una especie de fácil enraizamiento, ya que la probabilidad de enraizamiento sin aplicación de ANA fue del $35 \%$. No obstante, dicha probabilidad aumentó al doble con el uso de ANA $(\geq 1000$ mg.L $\left.\mathrm{L}^{-1}\right)$, sin afectar la calidad de dicho enraizamiento. Dado que no se detectaron diferencias entre concentraciones de ANA en cuanto a la probabilidad de enraizamiento, una dosis de 1000 ppm se considera sufi-

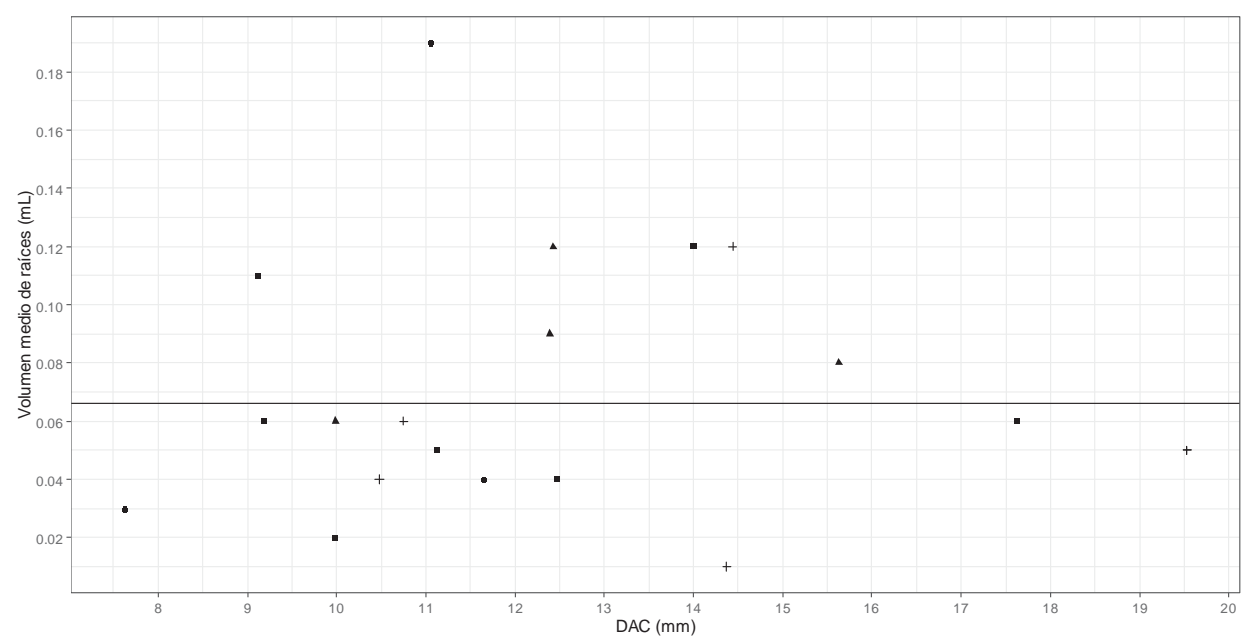

Figura 6. Volumen medio de raices en estacas de Croton urucurana. (•) sin aplicación de ANA, (৯) 1000 ppm de ANA, (ロ) 2500 ppm de ANA y (+) 3500 ppm de ANA.

Figure 6. Cutting diameter effect (DAC) on average root volume (ARV) on Croton urucurana

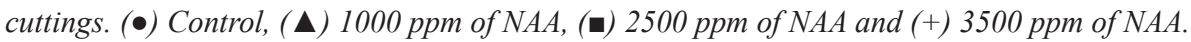
$A R V=0.066 \mathrm{~mL}$. 
ciente para promover el enraizamiento de estacas leñosas en C. urucurana. Desde el punto de vista comercial, es importante notar que la mayor longitud de raíces se obtuvo en estacas de aproximadamente $20 \mathrm{~mm}$ de diámetro basal, lo que indica que un diámetro superior a éste, sería el recomendado para la propagación asexual por estacas sin hojas.

Las estacas leñosas sin hojas de "Horquetero" (Tabernaemontana catharinensis A. DC.) no mostraron potencial para enraizar en las condiciones evaluadas. De acuerdo a los antecedentes bibliográficos, debería elevarse el tiempo de inmersión en ANA de 10 segundos a 10 minutos y evaluar estacas sub-leñosas con hojas.

Paullinia elegans Cambess ("Ojo de muñeca”) se comportó como una especie de fácil enraizamiento dado que se obtuvo una probabilidad de enraizamiento superior al 50\% en estacas sin hojas y con DAC superiores a $13 \mathrm{~mm}$. Desde el punto de vista comercial, nuestro estudio demuestra la factibilidad de la producción por estacas sin la aplicación de hormonas, utilizando estacas leñosas con diámetro mayor a 13 $\mathrm{mm}$. No obstante, restaría evaluar el efecto de la etiolización sobre el enraizamiento de estacas con DAC superior a $13 \mathrm{~mm}$ para la producción a nivel comercial.

\section{AGRADECIMIENTOS}

Este trabajo se desarrolló con fondos provenientes del proyecto $\mathrm{CAI}+\mathrm{D}$ Orientado a Problemas Sociales y Productivos, titulado "Introducción de especies nativas con potencial ornamental al mercado florícola", y del CAI $+\mathrm{D}$ "Aprovechamiento sustentable de productos forestales no madereros de especies nativas del Chaco argentino" (código 50020150100003LI), ambos financiados por la Universidad Nacional del Litoral.

Queremos agradecer al Dr. Agustín Alesso de la Cátedra de Estadística de la Facultad de Ciencias Agrarias de la Universidad Nacional del Litoral por su ayuda con el manejo de R.

\section{BIBLIOGRAFÍA}

Albertino SMF, Filho N, Do FJ, Silva JF da, Atroch AL, Galvão AK de L. Enraizamento de estacas de cultivares de guaranazeiro com adubação de plantas matrizes. Pesquisa Agropecuária Brasileira. 2012 Oct;47(10):1449-54.

Almeida FD de, Xavier A, Dias JMM, Paiva HN. Eficiência das auxinas (AIB e ANA) no enraizamento de miniestacas de clones de Eucalyptus cloeziana F. Muell. Revista Árvore. Sociedade de Investigações Florestais; 2007;31(3):455-63.

Amaya JZE, Yuyama K, Chagas EA, Fernández IM, Sakazaki RT, Neto JLLM. Management of Propagation Techniques of the Specie Croton lechleri Muell.Arg. Journal of Agricultural Science. 2019 May 15;11(6):486.

Amorim T de A, Coelho NL, Somner GV, Amorim T de A, Coelho NL, Somner GV. What is the Effect of Thiamine Hydrochloride on Rooting of Sapindaceae Stem Cuttings? Floresta e Ambiente [Internet]. Instituto de Florestas da Universidade Federal Rural do Rio de Janeiro; 2019 [cited 2020 Mar 4];26(3). Available from: http://www. scielo.br/scielo.php?script=sci_abstract\&pi$\mathrm{d}=$ S2179-80872019000300404\&lng=en\&n$\mathrm{rm}=$ iso\&tlng $=\mathrm{en}$ 
Arruda MR de, Pereira JCR, Moreira A, Teixeira WG. Enraizamento de estacas herbáceas de guaranazeiro em diferentes substratos. Ciência e Agrotecnologia. 2007 Feb;31(1):236-41.

Atroch AL, Cravo M da S, Santos JA dos. Enraizamento de estacas de clones de guaranazeiro tratados com ácido indol-3-butírico (AIB). Revista de Ciências Agrárias/Amazonian Journal of Agricultural and Environmental Sciences. 2011 May 9;47(1):103-12.

Chaves TDA. Propagação vegetativa de espécies florestais com potencial para uso como cerca viva [Tesis de Maestría]. [Seropedica, BRAZIL]: Universidade Federal Rural dDo Rio Dde Janeiro; 2016.

Cunha CSM, Maia SSS, Coelho M de FB. Estaquia de Croton zehntneri Pax et Hoffm. com diferentes concentrações de ácido indol butírico. Ciência Rural. Universidade Federal de Santa Maria; 2012 Apr;42(4):621-6.

da Gama L, Fernandes B, Yuyama K. Propagação vegetativa de sangue de dragão (Croton lechleri Müll. Arg.) por meio de estaquia no Estado do Amazonas. Annals of LXI (61a) Annual Meeting of the InterAmerican Society for Tropical Horticulture - ISTH. Brasilia, Brazil: EMBRAPA; 2015. p. 148.

de Carvalho J, Frazao D, Figueiredo F, Oliveira de R. Conservation of seed viability of guarana, Paullinia cupana var. sorbilis (Mart.) Ducke. Belem, Brasil: Centro de Pesquisa Agropecuaria do Tropico Umido; 1982.

Dias JPT, Takata WHS, Takahashi K, Ono EO. Propagação de figueira com estacas de diferentes diâmetros. Revista Trópica: Ciências Agrárias e Biológicas [Internet]. 2013 Aug 9 [cited 2020 Mar 16];7(1). Available from: http://www.periodicoseletronicos.ufma.br/ index.php/ccaatropica/article/view/863
Dole J, Gibson J. Cutting propagation: a guide to propagating and producing floriculture crops. 1st ed. Dole J, Gibson J, editors. Batavia, USA: Ball Publishing; 2006.

Flores que generan equidad [Internet]. INTA Informa. 2017 [cited 2020 Mar 17]. Available from: https://intainforma.inta.gob. ar $/ \%$ e $2 \% 80 \% 9$ cla-floricultura-genera-mas-de-us-200-m-anuales $\%$ e $2 \% 80 \% 9 \mathrm{~d} /$

Francis J. Wildland shrubs of the United States and its territories: Thamnic descriptions. IITF-GTR-26. San Juan, PR: U.S. Department of Agriculture, Forest Service, International Institute of Tropical Forestry; Fort Collins, CO: U.S. Department of Agriculture, Forest Service, Rocky Mountain Research Station.; 2004.

Ghio N, Buyatti M, Marinoni L, Biganzoli M, Gabriel P, Castro D. Evaluación del comportamiento germinativo de especies nativas con potencial ornamental del centro-norte santafesino. Horticultura Argentina. 2018;37(94):80.

Hartmann HT, Kester DE, Davies FT. Plant Propagation, principles and practice. 7 th ed. New Jersey, USA: Prentice Hall; 2002.

Hebbali A. olsrr: Tools for Building OLS Regression Models. [Internet]. 2018. Available from: https://CRAN.R-project.org/package $=$ olsrr

Kate KT, Laird SA. Biodiversity and Business: Coming to Terms with the 'Grand Bargain.' International Affairs. 2000;76(2):241-64.

Lenth R. emmeans: Estimated Marginal Means, aka Least-Squares Means [Internet]. 2020. Available from: https://CRAN.R-project.org/ package $=$ emmeans 
Martins JCR, Garrido M da S, Menezes RSC, Dutra ED, Primo DC, Jesus KN de. Desenvolvimento inicial de mudas de gliricídia e maniçoba preparadas com estacas de quatro comprimentos - DOI:10.5039/ agraria.v7i2a1533. Revista Brasileira de Ciências Agrárias (Agrária). 2015 Jun 30;7(2):322-7.

Mata D, Facciuto G, Botto F. Investigación científica y plantas ornamentales. Ciencia Hoy [Internet]. 2013 [citado 2020 Mar 17]. Disponible en: https://cienciahoy.org. ar/2013/12/investigacion-cientifica-y-plantas-ornamentales/

Ming LC. Rooting of cuttings of Lippia alba (Mill.) N.E.BR. - Verbenaceae. Acta Hortic. 1996 Aug;(426):643-6.

Morisigue, D. La floricultura genera más de U\$S $200 \mathrm{M}$ anuales. INTA Informa [Internet]. 2011 [citado 2020 Mar 17]. Disponible en: https:// intainforma.inta.gob.ar $\% \mathrm{E} 2 \% 80 \% 9$ Cla-floricultura-genera-mas-de-us-200-m-anuales $\% \mathrm{E} 2 \% 80 \% 9 \mathrm{D} /$.

Morisigue, D., 2017. Flores que generan equidad. INTA Informa. Año 15. No 171. ISBN 2362-5287.

Osakada A, Yuyama K. Propagação vegetativa de Sangue-de-dragão (Croton lechleri müll. Arg.) na Amazônia central. Anais do 59o Congresso Nacional de Botânica. Natal, Rio Grande do Norte. Brazil: Sociedade Botânica do Brasil; 2008. p. 1.

R Development Core Team. R: A Language and Environment for Statistical Computing [Internet]. Vienna, Austria: R Foundation for Statistical Computing; 2017. Available from: http://www.R-project.org

Rawat J, Singh K, Rawat V, Singh B. Effect of methods of IBA application on rooting performance of crape jasmine (Chandani) softwood cuttings. HortFlora Research Spectrum. 2014;3(2):146-9.
Richmond A. Estudio de cuatro métodos de propagación de cinco especies forestales. Universidad de Costa Rica, San José (Costa Rica) CATIE, Turrialba (Costa Rica); 1984 [cited 2020 Mar 16]; Available from: http:// repositorio.bibliotecaorton.catie.ac.cr:80/ handle/11554/4984

Rodrigues JELF, Lucchesi AA. Propagação vegetativa do guaranazeiro (Paullinia cupana (Mart.) Ducke) através de estacas induzidas (capeadas) e com ácido indolilbutirico. Anais da Escola Superior de Agricultura Luiz de Queiroz. 1987 Jan 1;44(1):1-20.

Santelices R. Efecto del árbol madre sobre la rizogénesis de Nothofagus alessandrii. Bosque (Valdivia). 2005 Dec;26(3):133-6.

Santos J de P dos, Davide AC, Teixeira LAF, Melo AJS, Melo LA de. Enraizamento de estacas lenhosas de espécies florestais. CERNE. Universidade Federal de Lavras; 2011 Sep;17(3):293-301.

Scalon SPQ, Mussury RM, Lima AA. Germination of Croton urucurana L. seeds exposed to different storage temperatures and pre-germinative treatments. Anais da Academia Brasileira de Ciências. Academia Brasileira de Ciências; 2012 Mar;84(1):191-200.

Silva LF de O da, Oliveira AF de, Pio R, Zambon CR, Oliveira DL. Enraizamento de estacas semilenhosas de cultivares de oliveira. Bragantia. 2012;71(4):488-92.

Soto MS, Greppi JA, Facciuto G. Exploration and Collection of Ornamental Germplasm Native to Argentina. Floriculture and Ornamental Biotechnology. 2011;5(1):10-22.

Wickham H. ggplot2: Elegant Graphics for Data Analysis [Internet]. Springer-Verlag New York,; 2016. Available from: https://ggplot2. tidyverse.org

WinRHIZO. Quebec, Canada: Regent Instruments; 2016. 